Action Report for a real-world response, this process can yield data that can be used to enhance ongoing response operations as well as to support anticipated response operations, such as applying lessons from one pandemic wave to the next. During the session, participants will be provided an opportunity to discuss their process for evaluating real-world events and to identify how this methodology can be integrated into their organization's response evaluation activities.

Prehosp Disaster Med 2011;26(Suppl. 1):s17-s18 doi:10.1017/S1049023X11000690

(A65) Stress \& Trauma Studies Program (STSP): Theoretical \& Practical Emergency Mental Health Interventions Studies for BA Social Work Students M.U. Farchi

School of Social Work, Stress \& Trauma Studies Program, Upper Galilee, Israel

The Tel Hai college Department of Social Work established this program as part of its community commitment to ensure that persons with skills in emergency mental health / trauma intervention will be available to the community as first responders when needed. The main goal of the STSP: Training Social work students As First Responders with Very High Professional Standards of Emergency as well as Long Term Mental Health Interventions Qualifications. This program enables the students to integrate between theory and hands-on basic and advanced skills in stress \& trauma interventions - from the help to a single traumatized person to mass disasters involving more complex interventions. In addition, program underlines and empowers the students self efficacy and resilience. The studies are carried out in 4 main channels: A. Academic studies and advanced professional workshops. B. Outdoor drills with other help and rescue units: MDA (EMS), IDF, Police, Israel fire and rescue services, local and national rescue units) C. Volunteering in community trauma / first responder units D. Emergency mental health interventions during real time events (Last one: Emergency interventions among the evacuated families during the mount Carmel bushfire) Student's Skills Acquired During the STSP • Theoretical $\&$ practical knowledge of the stress \& trauma development process. - Differentional diagnosis of the trauma stages (From ASR to C-PTSD). - Identifying all sources of resilience and coping strategies. - Basic \& advanced crisis and disaster intervention methods. $\bullet$ Crisis \& disaster management \& command - Professional self confidence, Independency \& Creativity, leadership and leading capabilities. The program, its benefits and latest drills and real time intervention will be discussed as well as demonstrated with videos.

Prehosp Disaster Med 2011;26(Suppl. 1):s18

doi:10.1017/S1049023X11000720

(A66) Integration of Psycho-Social Social Support and Mental Health Services in to National Disaster Management Guidelines

J.K. Christy

Psychosocial Support and Mental Health Services, New Delhi, India

Integration of Psycho-social Social Support and Mental Health Services in to National Disaster Management Guidelines India is vulnerable, in varying degrees, to a large number of natural as well as man-made disasters and also a high risk country for disasters due to expanding population, urbanization and industrialisation, development within high-risk zones, environmental degradation and climate changes. The creation of National Disaster Management Authority (NDMA) in 2005, as the apex body for disaster management, has brought out a paradigm shift in the area of disaster management. One of the important mandate of NDMA is to issue National Disaster Management Guidelines (NDMG) to the ministries/ departments to assist them to formulate their respective Disaster Management (DM) plans. In this direction NDMA has issued number of NDMG on different themes to provide basis of preparation of DM plans at different levels. There are policies \& guidelines on Psycho-social Support and Mental Health Services (PSSMHS) in disasters at the international level in the form of Inter Agency Standing Committee guidelines (IASC) which advocates PSSMHS in disasters. In India there was no such policy which streamlines the Psycho-social Support and Mental Health Services in Disasters. During preparation of various National Disaster Management Guidelines, one remarkable factor noticed was the need for psycho-social care, subsequently preparation of NDMG on Medical Preparedness and Mass Causality Management brought out an overwhelming consensus to formulate a separate NDMG for PSSMHS. In order to translate the critical need for psycho-social care and support into guidelines, NDMA adopted a mission-mode approach for integrating PSSMHS in disaster response by involving participatory and multi step methodology to formulate NDMG on Psycho-social Support and Mental Health Services in Disasters.

Prehosp Disaster Med 2011;26(Suppl. 1):s18

doi:10.1017/S1049023X11000732

\section{(A67) Empowerment Model for Community Disaster (EMCD)}

M.U. Farchi, ${ }^{1}$ E. Shahar ${ }^{2}$

1. School of Social Work, Stress \& Trauma Studies Program, Upper Galilee, Israel

2. School of Social Work, Upper Galilee, Israel

The earthquake in Haiti and its consequences highlighted the need to enhance knowledge and skills for community intervention in situations of disaster and acute trauma. The large number of homeless people concentrated in enormous refugee camps has made such an investigation possible. In the lecture, we will present a model for empowerment intervention with victims of community disasters. The model is based on analyses of three cases in which psychosocial interventions were conducted by the investigators: the Tsunami in Sri Lanka, work at refugee camps in Georgia, and the earthquake in Haiti. Principles of the Model The more extensive the casualties are, the less relevant individual intervention will be. Entering an unfamiliar culture requires collaborative professional work with local residents. Intervention in a large-scale disaster needs to be based on an interdisciplinary perspective in terms of planning, preparation, and implementation. It is assumed that the intervention will be short-term, and a specific length of time is allocated for therapeutic agents to provide assistance. This approach was adopted in light of the 
limited resources at our disposal, and in an attempt to minimize dependence in the relationships between the therapeutic agents and the victims. An attempt is made to enhance efficacy for effective coping with changing needs that emerge in the wake of the disaster. An attempt is made to prevent CPTSD, which can inhibit the functioning of the community residents. We will present these principles and describe how they were implemented in community intervention at two refugee camps in Haiti following the earthquake there, and at a refugee camp in Georgia.

Prehosp Disaster Med 2011;26(Suppl. 1):s18-s19

doi:10.1017/S1049023X11000744

(A68) 7 Options for Evolving the Concept of Disaster Health

A. Allen, ${ }^{1}$ J.M. Shultz, ${ }^{2}$ Z. Espinel ${ }^{3}$

1. Ace, Miami Shores, United States of America

2. Center for Disaster \& Extreme Event Preparedness, Miami, United States of America

3. Center for Disaster and Extreme Event Preparedness, Miami, United States of America

Introduction: The World Association for Disaster and Emergency Medicine (WADEM) has been the primary innovator and incubator for the concept of disaster health. This presentation puts forth 7 options for consideration for evolving the concept of disaster health.

Discussion: (1) Promote disaster health from an adjective to a noun phrase. Disaster health has been a modifier for too long, tucked into expressions such as disaster health management or disaster health education. It is time for disaster health to emerge in its own right. (2) Elevate disaster health from a discipline to an endpoint, a defined and desired outcome. (3) Liberate disaster health from the confines of medicine and health care. Disaster health originated there, but is much more expansive. One of the distinctions of disaster health is its multidisciplinary nature. (4) Fully integrate the mental health and psychological dimension of disaster health. WADEM has been at the forefront, championing psychosocial issues in disasters, yet to date, this dimension of disaster health has been underdeveloped and underappreciated. (5) Consider the parallels inherent in optimizing disaster health for both disaster responders and disaster survivors. Also consider using plain language to create a common set of strategies for achieving disaster health that is equally applicable for responders and survivors. (6) Consider disaster health applied at the community level in a manner that subsumes community health, resilience, and disaster resistance. (7) Give disaster health its own framework. Clear and comprehensive WADEM-driven frameworks now exist for disaster health education, for example. Disaster health needs a framework that is simple, supple, and explanatory.

Conclusion: WADEM has promulgated disaster health as a vital, pivotal concept. The 7 options presented here have come from our own engagement with this concept. They are, in fact, defining features of our SAFETY FUNCTION ACTION framework for disaster health.

Prehosp Disaster Med 2011;26(Suppl. 1):s19

doi:10.1017/S1049023X11000756
(A69) Epidemiological Study of Trauma in Pregnancy: An Emergency Department-Based Study of a Level-1 Trauma Center

G. Sharma, ${ }^{1}$ S. Bhoi, ${ }^{2}$ P. Gautam, ${ }^{3}$ G. Adhikari, ${ }^{3}$

D.T. Sinha, ${ }^{3}$ P. Manral, ${ }^{3}$ M. Rodha ${ }^{1}$

1. Emergency Department, New Delhi, India

2. Department Of Emergency Medicine, Trauma Centre, 110029, India

3. Emergency Medicine, Delhi, India

Background: Trauma during pregnancy poses a challenge in assessment and management due to its unique anatomical and physiological changes. Trauma is the leading non-obstetrical cause of death. There is paucity of epidemiological data in this subgroup in India. An emergency department (ED)-based epidemiological study was conducted.

Methods: Female trauma victims of reproductive age with both positive and negative urinary pregnancy tests (UPTs) were selected retrospectively. Documentation was done by the nursing staff from the ED case records. Mode, mechanism, severity, site of injury, and ED disposal time were noted, compiled, and analyzed.

Results: Of 64 patients, 32 patients were UPT-positive and 32 were UPT-negative. The mean age was 26 (range 18-36) years. A total of $75 \%$ of UPT-positive and $59.3 \%$ of UPT-negative cases had assault due to domestic violence. As per START triage protocol, $84.3 \%$ of UPT-positive and $59.3 \%$ patients in UPT negative were triaged as yellow. Blunt trauma to the abdomen was the most common mechanism and site of injury in all patients. FAST and ultrasonic evaluation of the fetus was performed for all UPT-positive patients. The average ED disposal time was 2 hours 62 minutes in UPT-positive and 1.9 hours in UPT-negative.

Conclusions: Limited data suggest domestic violence as leading cause of trauma in pregnancy. A large, epidemiological study is required to validate this.

Prehosp Disaster Med 2011;26(Suppl. 1):s19

doi:10.1017/S1049023X11000768

(A70) Traumatic Brain Injuries at a Rural Teaching Hospital: Pattern of Presentation and Documentation

A. Agrawal, ${ }^{1}$ A. Kakani, ${ }^{2}$ N. Baisakbiya, ${ }^{1}$

S. Galwankar, ${ }^{1}$ S. Dwivedi ${ }^{1}$

1. Neurosurgery, Ambala, India

2. Neurosurgery, Wardha, India

Background and Objectives: Analyses of causes and trends of traumatic brain injuries help to define public health policy priorities. There are not much TBI registries, thus making documentation of injuries inadequate and accessing these data problematic. This study is aimed at identifying the characteristics of TBI and determining the efficiency of documentation of patients' records in a tertiary hospital.

Patients and Methods: Based on WHO guidelines "Standards for Surveillance of Neurotrauma" we designed a proforma to collect data on traumatic brain injuries. A prospective data collection was done from January to June 2010. Data was collected on a paper form and then entered into the self-developed TBI registry database. Descriptive analysis was performed.

Results: Data for a total 414 patients were collected. Mean age was 33.00 years ( $\mathrm{SD} \pm 16.725$, range $1-85$ years), and $81 \%$ male. 